\title{
Volker Bauer (dir.), Frauen - Bücher - Höfe. Wissen und Sammeln vor 1800
}

\section{Claire Gantet}

\section{OpenEdition}

\section{Journals}

Édition électronique

URL : http://journals.openedition.org/ifha/10188

DOI : $10.4000 /$ ifha. 10188

ISSN : 2198-8943

\section{Éditeur}

IFRA - Institut franco-allemand (sciences historiques et sociales)

\section{Référence électronique}

Claire Gantet, « Volker Bauer (dir.), Frauen - Bücher - Höfe. Wissen und Sammeln vor 1800 », Revue de I'IFHA [En ligne], Date de recension, mis en ligne le 17 juillet 2019, consulté le 24 septembre 2020. URL : http://journals.openedition.org/ifha/10188; DOI : https://doi.org/10.4000/ifha.10188

Ce document a été généré automatiquement le 24 septembre 2020

(CIFHA 


\title{
Volker Bauer (dir.), Frauen - Bücher - Höfe. Wissen und Sammeln vor 1800
}

\author{
Claire Gantet
}

\section{RÉFÉRENCE}

Volker Bauer, Elizabeth Harding, Gerhild Scholz Williams, Mara R. Wade (dir.), Frauen Bücher - Höfe. Wissen und Sammeln vor 1800. Women - Books - Courts. Knowledge and Collecting before 1800. Essays in honor of Jill Bepler, Wiesbaden: Harrassowzitz Verlag in Komission (Wolfenbütteler Forschungen, 151), 2018, 451 p., $78 €$ 
Quiconque travaille sur le XVII ${ }^{\mathrm{e}}$ siècle va à Wolfenbüttel. Et quiconque a effectué des recherches dans la merveilleuse bibliothèque de Wolfenbüttel a connu Jill Bepler qui, jusqu'en juin 2018, dirigea le département des bourses, les manifestations et le soutien à la "relève " à la Bibliothèque du duc Auguste. On la connait non seulement comme organisatrice aussi discrète qu'efficace, notamment des pauses-café - des temps de sociabilité ainsi quasiment institutionnalisés dans la vie silencieuse des chercheurs plongés dans leurs lectures-mais aussi comme chercheuse spécialisée dans les voyages et les collections nobles, les textes publiés à l'occasion des funérailles, l'éducation dans

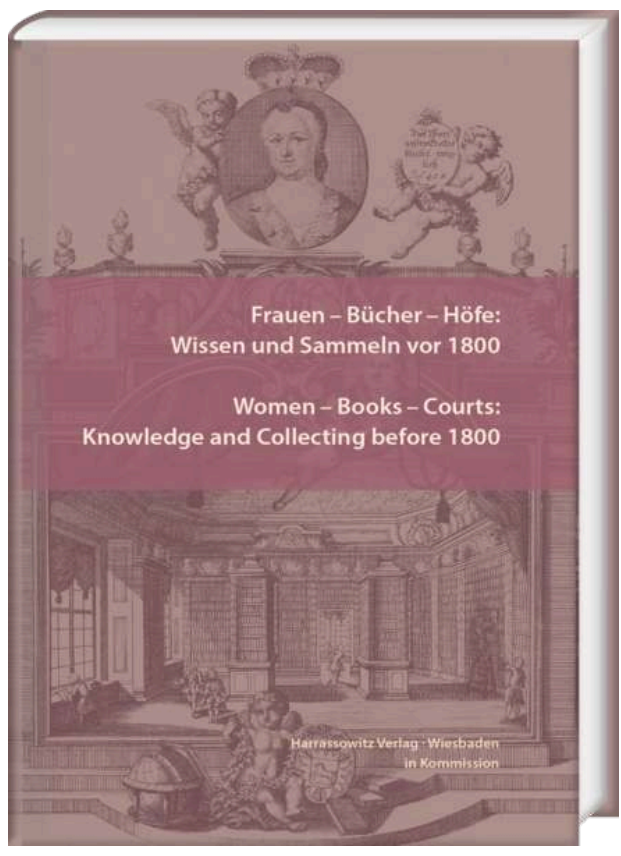
des cours allemandes et le rôle afférent de la princesse (que celle-ci soit reine, régente, épouse ou veuve), enfin les bibliothèques des princesses au XVII ${ }^{\mathrm{e}}$ siècle. Les recueils de mélanges auraient par excellence relevé des «Quodlibetica» (divers) qui constituaient non moins de 20400 titres des 135400 volumes détenus et répertoriés par le fondateur, le duc Auguste le Jeune (1579-1666). Or, les 29 collègues de Jill Bepler-des spécialistes de l'histoire des bibliothèques, de germanistique, d'histoire, de musicologie et de théologie - vont bien au-delà en développant un propos commun : manifester, au moyen d'études de cas dans le sillon des travaux de Jill Bepler, les perspectives ouvertes par le croisement des recherches sur l'histoire des cours, du genre et des collections.

Dès le milieu du XVII ${ }^{\mathrm{e}}$ siècle et en dépit de la guerre de Trente ans, la bibliothèque de Wolfenbüttel est la plus grande au nord des Alpes. Le duc Auguste le Jeune se stylisa en tant que lettré dans sa dynastie. Ulrich Johannes Schneider montre comment, au moyen notamment de sa roue à livres permettant de consulter simultanément plusieurs folios, il constitua 31300 recueils bibliographiques ordonnés par discipline (Theologia, Poetica, etc.). Leibniz eut le premier l'idée, lorsqu'il devint directeur de la bibliothèque (1691-1716), de leur substituer un catalogue par auteur, fondant par là une compréhension de la science comme production d'auteurs, un principe repris et amplifié par l'Historia literaria qui se développait concomitamment ; les listes d'auteurs s'ouvraient à la constitution de réseaux, et c'est à partir d'une telle liste, datée de 1699, que Leibniz projeta l'Académie des sciences de Berlin. Un tel principe, signale Ulrich Johannes Schneider, ne fut introduit qu'en 1776 à Göttingen, pourtant volontiers célébrée comme la bibliothèque la plus moderne de son temps.

Dans ce cadre, la place et le rôle des princesses doivent être réévalués. Partant de l'étude des cinq premières Utopies, Jörg Jochen Berns souligne qu'un certain nombre de lettrés dont les auteurs des premières utopies (Thomas More, Caspar Stiblin, Johann Valentin Andreae et Tommaso Campanella, voire Francis Bacon) « reconnaissaient aux femmes l'intelligence et l'aptitude à apprendre qui ne leur étaient pas accordées dans la réalité de l'Europe contemporaine » (p.143). Le rang de princesse n'offrait que peu de 
marges de liberté. Le cérémonial ne fonctionnait pas toujours (Thomas Rahn), leur rôle se limitait à la cour - à l'exclusion de l'administration et de l'armée (Volker Bauer) - et il leur fallait parfois se battre pour bénéficier d'un héritage (Helga Meise). Les hommes nobles toutefois, relève Gabriele Ball, disposaient certes des savoirs acquis lors de leur Grand Tour, mais fréquentaient rarement l'université et possédaient encore plus exceptionnellement un diplôme. Partout, comme l'a démontré Helga Meise, le moi individuel tendait à s'effacer devant les besoins de représentation. Ainsi, par exemple, les sermons funèbres pour les princesses-électorales de la dynastie Hohenzollern, convertie au calvinisme en 1613, étaient-ils empreints d'un irénisme destiné à rassembler, face au territoire resté massivement luthérien comme aux princes extérieurs (Sara Smart). Dans ces limites bien entendues, certaines femmes, bien entourées, purent jouer un rôle essentiel dans la vie culturelle et intellectuelle de leur cour.

Ulrike Gleixner rappelle en effet que la plupart des princesses possédaient une bibliothèque privée qu'elles augmentèrent, une fois mariées, par des acquisitions ou des dons ; elle servait des fins d'affirmation politique, dynastique ou confessionnelle, ainsi que des pratiques de piété et d'écriture. À la cour d'Anhalt de confession réformée, dans la première moitié du XVII ${ }^{e}$ siècle, les princesses étaient savantes, maîtrisant le latin comme le français, témoignant d'intérêts et de compétences littéraires et musicaux de même que de connaissances médicales et pharmaceutiques (Andreas Herz). Mara R. Wade se penche plus précisément sur le livre d'emblèmes que Dorothea d'Anhalt, princesse de Brunswick-Lunebourg, annota et fit signer par des nobles d'autres cours. Étudiée par Cornelia Niekus Moore, Sibylle Ursula de BrunswickLunebourg était une musicienne et compositrice talentueuse; elle possédait une bibliothèque privée de 260 volumes et écrivit elle-même, notamment, une première version du roman Aramena, publié ultérieurement par son frère Antoine-Ulrich. La princesse Christiane zu Waldeck-Pyrmont, présentée par Helga Meise, disposait elle aussi d'une bibliothèque de presque 200 livres (dont 4 seulement de théologie et plus de la moitié d'« histoires»!). L'exemple le plus fort est sans doute celui de Sophia de Anhalt-Köthen née comtesse de Lippe (1599-1654), exposé par Gabriele Ball. Peu après son mariage avec le prince Louis d'Anhalt-Köthen en 1626, elle intégra la Tugendliche Gesellschaft (Société vertueuse), qui réunissait 103 femmes protestantes de la haute noblesse, et la Noble Académie des Loyales, une société féminine appelée à fonctionner comme un réseau dynastique féminin. Son mari dirigeait la Société Fructifère (Fruchtbringende Gelsellschaft), la première grande société littéraire allemande de 1617 à 1680 , sous le nom du Nourricier. Avec l'appui de son mari, elle intégra le cœur de la Société, cosigna des textes fondateurs et fut dénommée la Nourricière.

Même si les volumes allemands ne sont habituellement pas garnis de conclusion (il revient au lecteur de s'en faire une), des mots conclusifs auraient conféré plus de force au propos en présentant un véritable programme de recherche. Les termes du titre du recueil sont en effet conçus avant tout comme un dénominateur commun à des études réunies pour l'occasion. On regrette en particulier l'absence de problématisation du "savoir» (Wissen/Knowledge) et des "collections" (Sammeln/Collecting), très inégalement discutés au gré des différentes contributions. En dépit de cette faiblesse, le volume manifeste la fertilité du croisement de l'histoire matérielle des savoirs et de l'histoire culturelle des cours ouverte à la problématique du genre, et l'on sait gré à jill Bepler d'avoir engagé de telles recherches. 
INDEX

Thèmes : Histoire des femmes, histoire du genre

Index chronologique : Période moderne

\section{AUTEURS}

CLAIRE GANTET

Université de Fribourg (Suisse)/Universität Freiburg (Schweiz) 